\title{
American Society of Hematology 2020 Podcast Collection: CML
}

\author{
Massimo Breccia
}

Received: February 3, 2021 / Accepted: February 10, 2021 / Published online: March 15, 2021

(C) The Author(s) 2021

\section{PODCAST TRANSCRIPT}

Victoria Glasson (VG): Managing Editor of Advances in Therapy.

Massimo Breccia (MB): Department of Translational and Precision Medicine, University of Rome.

VG: Welcome to the Adis Rapid+ Podcast Series. We're bringing you a selection of podcasts covering the American Society of Hematology 2020 Conference, discussing the highlights of the data released. Today's podcast will be focusing on chronic myeloid leukaemia and the data presented at the ASH Conference.

Speaking to us today is Dr. Massimo Breccia, Department of Translational and Precision Medicine at the Sapienza University of Rome. Massimo, welcome to today's podcast, and thank you so much for speaking with us. You're going to take us through your top highlights of the CML [chronic myeloid leukaemia] data from the ASH 2020 Conference, and lots of important data was released. And to get us started, what would be the first highlight of the data that you would like to discuss?

MB: So thank you very much, at first, for this kind invitation, and I would like to start with a

\section{Breccia $(\square)$}

Department of Translational and Precision

Medicine, University of Rome, Rome, Italy

e-mail: breccia@bce.uniroma1.it new drug. Asciminib, the first example of allosteric inhibition, now called STAMP inhibitor from specifically targeting the ABL myristoyl pocket. So the first oral communication was about the use of this drug in a phase I study in the expanded cohort that used the dose of $200 \mathrm{mg}$ BID for patients with CML and T315I mutation [1]. 52 patients were enrolled in this expansion cohort. Of them, more than 30\% were previously pretreated with three or more TKIs, and 31 received ponatinib. 35 patients remain in treatment, and the median duration of exposure was 68 weeks.

And in terms of efficacy, it was reported that the major molecular response (MMR) rate was $57.8 \%$ in ponatinib-naive patients, compared to $28.6 \%$ in ponatinib-pretreated patients. So even the MR4 and the MR4.5 rates were higher for patients ponatinib-naive and obtained at median of 20 weeks. As to safety, even the increased doses of the drug were not associated to a different safety profile they reported previously in the phase I study, and the most common adverse events, in fact, were fatigue, nausea, diarrhoea, and increased, but reversible lipase. Only three patients experienced arterial occlusive events.

And asciminib was also the main actor of another oral communication presented as at the late-breaking abstract by Professor Hochhaus that reported for the first time the results of the 
phase III trial called ASCEMBL that compared asciminib, $40 \mathrm{mg}$ BID, to bosutinib, $500 \mathrm{mg}$ per day in chronic phase patients that were previously pretreated with two lines of treatment [2]. And the main inclusion criterion is that the molecular ratio at the time of enrolment should be higher than $0.1 \%$. The primary endpoint of this trial was the achievement of the major molecular response at 24 weeks; 220 patients were enrolled, and 97 patients remain in treatment in the asciminib arm. A switch was also allowed a switch from bosutinib to asciminib in case of resistance and intolerance.

This strategy was performed, in fact, in 22 patients that were enrolled at baseline to the bosutinib arm. The primary endpoint was reached because the MMR rate was $25.5 \%$ in the asciminib arm compared to compared to $13.2 \%$ in the bosutinib arm, and the median time to achieve this response is similar, 12.7 weeks for asciminib and 14.3 weeks for bosutinib.

So even the deep molecular response, in terms of MR4 and MR4.5 rates were in favour of asciminib, and the responses were reported higher in the asciminib arm, regardless of baseline future. The most frequent side effects recorded in the asciminib arm were thrombocytopenia and neutropenia, whereas no new safety events with bosutinib because the main adverse events recorded were diarrhoea and liver impairment with an increased transaminase level. So I believe that according to this data presented for asciminib, this drug probably will change our treatment strategy in the near future, in particular in third line-but not only, probably, even in earlier line-and could be also another important drug for patients with T315I mutation, for example, for the subset of patients that cannot receive ponatinib for the concomitant cardiovascular comorbidities.

Another important final analysis was reported at the last ASH meeting, the Bfore trial that enrolled one to one 536 patients randomized to bosutinib $400 \mathrm{mg}$ and the standard dose imatinib, newly diagnosed patients [3], and the final analysis is at 5 years of follow-up. So at the final analysis, more than half of the patients remain in treatment in both arms, and all the molecular response rates were in favour of bosutinib, from MMR to the deep MR4.5. Even for the early molecular response achievement at 3 months, that is $80.6 \%$ in the bosutinib arm, as compared to $60.5 \%$ for the standard dose imatinib, where it also reported more, faster responses in the bosutinib arm, even in high Sokal risk patients. And the number of patients that experienced the blast crisis progression is the same from bosutinib to imatinib, six patients versus seven patients, but it has been reported the cumulative incidence of progression of death was in favour of bosutinib. In the bosutinib arm, more patients discontinued due to the adverse events, as comparing to the imatinib arm where more discontinued for the lack of efficacy. And again, the more common adverse events reported with bosutinib were gastrointestinal, diarrhoea, nausea, and increased transaminases.

So the final analysis of Bfore trial confirmed that bosutinib was superior to imatinib as a possible first-line standard of care, expected results as compared to also the other secondgeneration TKIs [tyrosine kinase inhibitors], dasatinib and nilotinib. And in fact, as in the ENESTnd and DASISION trials, no differences were reported in terms of overall survival as compared to standard dose imatinib. So the safety is consistent with the non-mid-term profile of the drug, and the gastrointestinal events, in fact, should be considered, I believe, as a specific profile of the drug in the frontline decision-making process.

For the first time, in another oral communication, the Japanese group reported the results of a trial called CML212 that compared for the first time in newly diagnosed patients, nilotinib versus dasatinib [4]; 461 patients enrolled that were stratified according to the Sokal risk. The primary endpoint was the achievement of a MR4.5 at 18 months.

Not surprisingly, the results showed that the drugs are equally effective because the MR4.5 rate was $32 \%$ with nilotinib and $31 \%$ with dasatinib. And also, no differences were revealed in terms of progression-free, event-free, and overall survival, and the safety profile was similar to what was reported before for the two drugs. In particular, increased lipase level for nilotinib and thrombocytopenia with dasatinib. So, the results showed that is not possible to 
discriminate the best frontline treatment only choosing one drug over another, but only according to the so-called patient-centred approach. So, considering other futures at baseline, in particular, age, comorbidities, and prognostic stratification based on prognostic scores, the most used now is the EUTOS longterm survival score, as even suggested by the international guidelines.

VG: That's really interesting, Massimo. Can I just say-so when really looking at using precision medicine when deciding treatment for a patient, it should be personalized for their circumstances and their comorbidities, rather than the decision being made on the drug itself?

MB: This process of the patient-centred approach started in Europe, so it was called, before, holistic approach because we consider all the possible clinical-related factors. As you know, CML has only the translocation 9;22 as a biological event, but no other markers of disease. So the first-line treatment was decided according to these clinical futures at baseline and also, for example, if the patients had some social and other characteristics that may impact on long-term adherence.

And in particular, in younger patients now, if this final endpoint should be the discontinuation of the treatment, the so-called treatmentfree remission. Most important, in fact, the comorbidities profile at baseline, the concomitant drugs and the scores. So, for example, for patients with high Sokal risk or ELTS high score, we prefer the second-generation TKIs.

VG: Responses in this trial are higher than in the ENESTnd and DASISION trials. Why do you think that is?

MB: So probably depends also from the geographical area in which the trial was performed. As you know, the Chinese population had a low BMI, the body mass index, that may influence the response rate with second-generation TKIs. And also, specific safety profile that, for example, is not the same in Europe or in the USA, which there is an increased rate of cardiovascular events that, for example, in the Chinese population or in the Japanese population was never reported.

Another new drug was presented by the Chinese group, before it was called HQP1351.
Now, the name is olverembatinib. The Chinese group tested this drug in two different trials, all involving patients with T315I mutation [5]. The CC201 study enrolled 41 patients in chronic phase.

More than half of the patients received previously two lines of therapy, but not ponatinib, so all the patients enrolled in this trial were ponatinib-naive. The complete cytogenetic response rate was $66 \%$, and the major molecular response rate in chronic phase was $48.8 \%$. And the safety profile was specific for this drug because more than $70 \%$ of patients experienced thrombocytopenia, and a peculiar skin pigmentation, and also liver impairment.

The CC202 study, indeed, used the drug in accelerated phase, and 23 patients were enrolled. So the complete cytogenetic response rate in this subset was 39\%, and the major molecular response rate was $26 \%$. Even in this subset, the thrombocytopenia was the main event because $74 \%$ of patients experienced hematological toxicity, skin pigmentation, but also some lab abnormalities, such as hypocalcaemia or hypertriglyceridaemia. So olverembatinib is another possible option for the subset of patients with T315I mutation, but for the moment, only for the Asiatic countries. This specific safety profile, I believe that narrows its field of action to a specific population that required more attention for dermatological toxicities.

The other one is an oral communication in which it was reported a pooled analysis of patients in chronic phase that received ponatinib [6]. More than 200 patients from the PACE trial and 92 from the OPTIC trial. So, in this latest trial, the patients were initially randomized to three different doses of ponatinib, 45, 30 , and $15 \mathrm{mg}$, but with the possibility to reduce the dose after the achievement of the primary endpoint. That is, the molecular ratio less than $1 \%$ after 1 year of treatment.

So, this endpoint was reached by $45 \%$ of patients in the PACE trial and 51.6\% in the OPTIC trial, and even the 2-year overall survival was in favour of the OPTIC trial and, in particular, the cardiovascular profile. Reducing the dose, it seems to be lower in the OPTIC trial as compared to the PACE trial. So to prove this, a 
propensity score was determinate between the two cohorts and, in fact, showed that the cardiovascular rate is $64 \%$ lower in the OPTIC trial as compared to the PACE. This analysis was based on the preliminary data of the OPTIC trial that were previously reported at the ASCO [American Society of Clinical Oncology] meeting. It seems that the strategy of reduction to $15 \mathrm{mg}$ in case of achievement of the primary endpoint seems to be the best strategy to reduce the number of cardiovascular events that probably can be followed in the future.

VG: So what do you think of the use of asciminib in combination therapies with TKIs in the future?

MB: So the drug was created to be combined because, as you know, it acts on the myristoyl pocket and not in the ATP site; that was the specific point of binding of all the other TKIs available. Some data were previously reported for the combination inside a phase I trial, but we have no data completed, and the trial is still ongoing for this part, but it seems a possible option, in particular because in vitro, the combination showed that it may prevent resistance to one TKI. So the first example in vitro was, in fact, combining nilotinib plus asciminib. And it seems also that, even in vitro, combined to ponatinib it acts also against compound mutations, that's the worst form of the mutation that we have.

VG: Well, that is a fantastic roundup of all of the CML highlights from ASH 2020. Thank you so much, Massimo, for joining us today. I hope this will be helpful for all of our listeners, and please look out for the other podcasts in this ASH 2020 Coverage Collection.

You can listen to more podcasts by subscribing to Adis Rapid+ podcast with your preferred podcast provider, or by visiting the website. A full list of declarations, including funding and author disclosure statements, can also be found on the journal website

\section{DIGITAL FEATURES}

This article is published with digital features, including a podcast video and audio file, to facilitate understanding of the article. To view digital features for this article go to https://doi. org/10.6084/m9.figshare.13701850.

\section{ACKNOWLEDGEMENTS}

Funding. This podcast has been developed independently through an unrestricted educational grant by Novartis. The Rapid Service Fee was funded by an unrestricted educational grant by Novartis.

Authorship. The named author meets the International Committee of Medical Journal Editors (ICMJE) criteria for authorship for this article, takes responsibility for the integrity of the work as a whole, and has given approval for this version to be published.

Disclosures. Massimo Breccia is on the Editorial Board of Advances in Therapy but has nothing further to disclose.

Compliance with Ethics Guidelines. This article does not contain any studies with human participants or animals performed by the author.

Peer Review. Please note, contrary to the journal's standard single-blind peer review process, as a podcast this article underwent review by a member of the journal's Editorial Board.

Open Access. This article is licensed under a Creative Commons Attribution-NonCommercial 4.0 International License, which permits any non-commercial use, sharing, adaptation, distribution and reproduction in any medium or format, as long as you give appropriate credit to the original author(s) and the source, provide a link to the Creative Commons licence, and indicate if changes were made. The images or other third party material in this article are included in the article's Creative Commons licence, unless indicated otherwise in a credit line to the material. If material is not included in the article's Creative Commons licence and your intended use is not permitted by statutory 
regulation or exceeds the permitted use, you will need to obtain permission directly from the copyright holder. To view a copy of this licence, visit http://creativecommons.org/licenses/by$\mathrm{nc} / 4.0 /$.

\section{REFERENCES}

1. Cortes KE, Hughes TP, Mauro MJ, et al. Asciminib, a first-in-class STAMP inhibitor, provides durable molecular response in patients (pts) with chronic myeloid leukemia (CML) harboring the T315I mutation: primary efficacy and safety results from a phase 1 trial. In: ASH 2020 Conference. 2020. https://ash. confex.com/ash/2020/webprogram/Paper139677. html.

2. Hochhaus A, Boquimpani C, Rea D, et al. LBA-4 Efficacy and safety results from ASCEMBL, a multicenter, open-label, phase 3 study of asciminib, a firstin-class STAMP inhibitor, vs bosutinib (BOS) in patients (Pts) with chronic myeloid leukemia in chronic phase (CML-CP) previously treated with $\geq 2$ tyrosine kinase inhibitors (TKIs). In: ASH 2020 Conference. 2020. https://ash.confex.com/ash/2020/ webprogram/Paper143816.html.
3. Brümmendorf T, Cortes JE, Milojkovic D, et al. Bosutinib (BOS) versus imatinib for newly diagnosed chronic phase (CP) chronic myeloid leukemia (CML): final 5-year results from the bfore trial. In: ASH 2020 Conference. 2020. https://ash.confex.com/ ash/2020/webprogram/Paper137393.html.

4. Matsumura I, Ohtake S, Atsuta Y, et al. Nilotinib vs. dasatinib in achieving MR4.5 for newly diagnosed chronic myeloid leukemia: results of the prospective randomized phase 3 study, JALSG CML212. In: ASH 2020 Conference. 2020. https://ash.confex.com/ash/ 2020/webprogram/Paper134168.html.

5. Jiang Q, Huang X, Chen Z, et al. Novel BCR-ABL1 tyrosine kinase inhibitor (TKI) HQP1351 (olverembatinib) is efficacious and well tolerated in patients with T315I-mutated chronic myeloid leukemia (CML): results of pivotal (phase II) trials. In: ASH 2020 Conference. 2020. https://ash.confex.com/ash/ 2020/webprogram/Paper142142.html.

6. Kantarjian H, Deininger MW, Abruzzese E, et al. Efficacy and safety of ponatinib (PON) in patients with chronic-phase chronic myeloid leukemia (CP$\mathrm{CML}$ ) who failed one or more second-generation (2G) tyrosine kinase inhibitors (TKIs): analyses based on PACE and Optic. In: ASH 2020 Conference. 2020. https://ash.confex.com/ash/2020/webprogram/ Paper133922.html. 